\title{
A role for dynamic contrast-enhanced magnetic resonance imaging in predicting tumour radiation response
}

\author{
Rami R Hallac ${ }^{1,2}$, Heling Zhou ${ }^{1}$, Rajesh Pidikiti ${ }^{3,6}$, Kwang Song ${ }^{3,7}$, Timothy Solberg ${ }^{3,8}$, Vikram D Kodibagkar ${ }^{1,4}$, \\ Peter Peschke 5 and Ralph P Mason*,1 \\ ${ }^{1}$ Department of Radiology, UT Southwestern, 5323 Harry Hines Boulevard, Dallas, TX 75390, USA; ${ }^{2}$ Analytical Imaging and \\ Modeling Center, Children's Medical Center, Dallas, 1935 Medical District Dr, Dallas, TX 75235, USA; ${ }^{3}$ Radiation Oncology, UT \\ Southwestern, 5323 Harry Hines Boulevard, Dallas, TX 75390, USA; ${ }^{4}$ School of Biological and Health Systems Engineering, Arizona \\ State University, Tempe, AZ 85278, USA and ${ }^{5}$ Department of Molecular Radiation Oncology, German Cancer Center, Im \\ Neuenheimer Feld 280, Heidelberg 69121, Germany
}

Background: Dynamic contrast-enhanced (DCE) MRI may provide prognostic insights into tumour radiation response. This study examined quantitative DCE MRI parameters in rat tumours, as potential biomarkers of tumour growth delay following single high-dose irradiation.

Methods: Dunning R3327-AT1 prostate tumours were evaluated by DCE MRI following intravenous injection of Gd-DTPA. The next day tumours were irradiated (single dose of $30 \mathrm{~Gy}$ ), while animals breathed air $(n=4)$ or oxygen $(n=4)$; two animals were nonirradiated controls. Growth was followed and tumour volume-quadrupling time $\left(T_{4}\right)$ was compared with pre-irradiation DCE assessments.

Results: Irradiation caused significant tumour growth delay ( $\mathrm{T}_{4}$ ranged from 28 to 48 days for air-breathing rats, and 40 to 75 days for oxygen-breathing rats) compared with the controls ( $T_{4}=7$ to 9 days). A strong correlation was observed between $T_{4}$ and extravascular-extracellular volume fraction $\left(v_{\mathrm{e}}\right)$ irrespective of the gas inhaled during irradiation. There was also a correlation between $T_{4}$ and volume transfer constant $\left(K^{\text {trans }}\right)$ for the air-breathing group alone.

Conclusions: The data provide rationale for expanded studies of other tumour sites, types and progressively patients, and are potentially significant, as many patients undergo contrast-enhanced MRI as part of treatment planning.

Tumours exhibit a range of response to therapy and there is an increasing desire to develop prognostic biomarkers to predict therapeutic efficacy. Proteomic and genomic screens may be related to tumour aggressiveness, but are often based on invasive biopsy specimens. Imaging potentially offers entirely non-invasive insight into tumour pathophysiology, which may influence tumour development and response to therapy.

Hypoxia is thought to influence radiation response and there have been many clinical trials aimed at exploiting or overcoming hypoxia (Wilson and Hay, 2011; Horsman et al, 2012). However, the meta-analysis of Overgaard (2007) suggested only a minor benefit for procedures designed to overcome hypoxia. There is general consensus that such trials are hampered by inability to identify hypoxic tumours and stratify patients for optimal therapy. This has led to extensive interest in developing biomarkers of hypoxia (Tatum et al, 2006). Perhaps the most effective, to date, has been the Eppendorf Histograph, which was successfully used to stratify human tumours at various disease sites based on various

\footnotetext{
*Correspondence: Professor RP Mason; E-mail: Ralph.Mason@UTSouthwestern.edu

${ }^{6}$ Current address: Department of Radiation Oncology, Willis-Knighton Health System, Shreveport, LA, USA.

${ }^{7}$ Current address: Texas Oncology, Fort Worth, TX, USA.

${ }^{8}$ Current address: Department of Radiation Oncology, University of Pennsylvania, Philadelphia, PA, USA.
}

Revised 25 February 2016; accepted 22 March 2016; published online 3 May 2016

(c) 2016 Cancer Research UK. All rights reserved 0007-0920/16 
oxygen-related criteria such as hypoxic fraction, median $\mathrm{pO}_{2}$ or ratio of tumour to local normal tissue (Falk et al, 1992; Fyles et al, 1998; Rofstad et al, 2000; Sheridan et al, 2000; Movsas et al, 2002; Le et al, 2006; Vaupel and Mayer, 2007). However, electrode application is highly invasive and is seldom used today. Hypoxia may also be identified histologically based on bioreductive markers such as pimonidazole, and this strategy was successfully applied in head and neck cancer (Kaanders et al, 2004). Ideally, stratification would be achieved non-invasively and indeed multiple strategies are being developed using MRI, ESR, optical and radionuclide imaging (Tatum et al, 2006; Mason et al, 2010; Fleming et al, 2015), although to date, none has been approved for routine clinical use.

Several studies have suggested that DCE MRI may provide insights into tumour hypoxia and radiation response, as well as perfusion (Zahra et al, 2007; Horsman et al, 2012), and correlations have been shown with electrode measurements and radiobiological hypoxic fractions (Lyng et al, 2001; Loncaster et al, 2002; Wang et al, 2002; Egeland et al, 2012). Hitherto, such studies have generally fallen into two classes: (i) pre-clinical animal investigations with quantitative DCE analysis, but in many cases assessment of radiation response was limited to paired clonogenic survival assays (Gulliksrud et al, 2011; Egeland et al, 2012; Ovrebo et al, 2012); (ii) clinical and large animal studies of response, generally relating semi-quantitative analysis of DCE based on relative signal enhancement, or initial area under the curve (Lyng et al, 2001; Loncaster et al, 2002; Mayr et al, 2009; Viglianti et al, 2009). Contrary results have been reported with more or less correlation between specific DCE parameters and hypoxia (Ovrebo et al, 2012). As such, we decided to include DCE MRI as part of a larger MRI investigation of oxygen-modulated tumour response to radiation. We have published the oxygen-enhanced (OE) MRI previously (Hallac et al, 2014), and now present the more complex analysis of DCE MRI.

Contrast-enhanced MRI is a routine clinical scan used to examine many tumour disease sites. It requires the administration of small molecular weight gadolinium (Gd) chelates that can aid in tumour delineation by virtue of the highly fenestrated leaky vasculature. Beyond the assessment of temporal and spatial variation in signal intensity following the administration of gadolinium, this technique has been widely used to evaluate tumour pathophysiology (Evelhoch, 1999; Tofts et al, 1999; Alonzi et al, 2007; Eyal and Degani, 2009). Sophisticated pharmacokinetic analysis has been developed initially by direct analogy with radiotracer methods (Tofts, 1997; Tofts et al, 1999). Several mathematical models, such as the modified Kety/Tofts model (Tofts et al, 1999), have been established to generate parametric images of volume transfer constant $\left(K^{\text {trans }}\right)$ and extravascularextracellular volume fraction $\left(v_{\mathrm{e}}\right)$.

In this study, we evaluated the potential use of DCE MRI for predicting the radiation response of the Dunning prostate R3327AT1 rat tumour. The day following MR imaging, tumours were irradiated with a single high dose of $30 \mathrm{~Gy}$ and growth delay was compared with pre-irradiation DCE assessments. These tumours formed a subset of a larger cohort evaluated previously for OEMRI. At the time, we were unable to evaluate the DCE MRI and therefore this study is presented separately here, in the context of the previous work (Hallac et al, 2014).

\section{MATERIALS AND METHODS}

Animal model. This study was approved by the UT Southwestern Institutional Animal Care and Use Committee in accordance with Federal, State, and Local laws and guidelines and consistent with Guidelines for the welfare and use of animals in cancer research (Workman et al, 2010). Ten male Copenhagen rats (weight
200 g) were implanted with Dunning R3327-AT1 rat prostate tumours. The AT1 is an anaplastic tumour with a volume-doubling time of 5.2 days and a $\mathrm{TCD}_{50}$ (single radiation dose for $50 \%$ tumour control probability) of $75.7 \mathrm{~Gy}$ (Peschke et al, 1998, 2011). Tumours were originally provided by Dr J.T. Isaacs of Johns Hopkins University (Isaacs et al, 1986). Donor tissue was grown in the thigh of Copenhagen rats and fresh tumour tissue fragments were collected and surgically implanted subcutaneously in the thigh via a small incision. Tumour volume was measured using a pair of calipers and calculated as $\mathrm{V}=(\pi / 6) \times \mathrm{a} \times \mathrm{b} \times \mathrm{c}$, where $\mathrm{a}, \mathrm{b}$ and $c$, are the three orthogonal diameters. When tumours reached about $0.3 \mathrm{~cm}^{3}$ they were evaluated by MRI and irradiated.

MRI. MRI used a horizontal bore 4.7-T magnet system (Agilent, Palo Alto, CA, USA) with home-built $2 \mathrm{~cm}$ single-turn solenoid volume coil. Animals were anaesthetised with isoflurane (1.5\%) in air $\left(1 \mathrm{dm}^{3} \mathrm{~min}^{-1}\right)$ and kept warm $\left(37^{\circ} \mathrm{C}\right)$ using a circulating warm water blanket. High-resolution $\mathrm{T}_{2}$-weighted images were acquired using a Fast Spin-Echo (FSEMS) sequence: TR/TE $\mathrm{eff}=2000 / 48 \mathrm{~ms}$, echo train length $(\mathrm{ETL})=8, \mathrm{FOV}=40 \times 40 \mathrm{~mm}$, acquisition matrix $128 \times 128$, NEX $=8,1 \mathrm{~mm}$ slice thickness without gap, and 15 slices acquired in the axial orientation. Interleaved BOLD (blood oxygen level dependent) and TOLD (tissue oxygen level dependent) (IBT) MRIs were acquired in an automated fashion using a home-written macro during air breathing followed by oxygen, as described in detail previously (Hallac et al, 2014). Following about $30 \mathrm{~min}$ oxygen breathing, quantitative $R_{1}$ measurements were acquired with $\mathrm{O}_{2}$-breathing using a spin-echo sequence (SEMS): $\mathrm{TE}=20 \mathrm{~ms}, \mathrm{TR}=0.1,0.2,0.3,0.5,0.7,0.9,1.5$, $2.5,3.5 \mathrm{~s}, \mathrm{FOV}=40 \times 40 \mathrm{~mm}^{2}$ with $64 \times 64$ acquisition matrix. Time course DCE data were acquired continuously using a SEMS with TR/TE of $200 / 15 \mathrm{~ms}$ providing $11 \mathrm{~s}$ temporal resolution preand up to $30 \mathrm{~min}$ post-injection of $0.1 \mathrm{mmol} \mathrm{kg}{ }^{-1}$ body weight $\mathrm{Gd}$ DTPA (Magnevist) intravenously in the tail vein rapidly by hand. Images were acquired with the same spatial resolution as $\mathrm{R}_{1}$ maps. $\mathrm{R}_{1}$ maps were obtained before contrast injection in order to facilitate conversion of signal-intensity data to contrast agent concentrations.

Radiation treatment. Radiation was delivered using a dedicated small animal X-ray irradiator (XRAD 225Cx, Precision X-Ray, Inc., North Branford, CT, USA). The XRAD 225Cx features an X-ray tube mounted on a c-arm gantry, which rotates about a motorised $\mathrm{x}-\mathrm{y}-\mathrm{z}$ (3D) translational stage (Parker 404XE, Parker-Hannifin Corp., Irwin, PA, USA). Absolute-dose calibration was performed in accordance with the recommendations of the AAPM TG-61 protocol ( $\mathrm{Ma}$ et al, 2001). The XRAD 225Cx was operated at $225 \mathrm{kV}$ and $13 \mathrm{~mA}$, producing a dose rate of $\sim 3.5 \mathrm{Gymin}^{-1}$. A circular aperture of $20 \mathrm{~mm}$ in diameter was used to limit the radiation beam only to the tumour. An image-guidance system utilising a digital-imaging panel for digital radiography, fluoroscopy and cone-beam CT was used to ensure accurate target localisation.

Rats were divided into three groups: Group 1 control $(n=2$, no irradiation); Group $2(n=4)$ breathed air during irradiation and Group $3(n=4)$ breathed oxygen 15 min before and during irradiation. Half the radiation dose $(15 \mathrm{~Gy})$ was delivered DorsalVentral, which was followed immediately by another dose of $15 \mathrm{~Gy}$ from the opposite side (Figure 1). Following irradiation tumour volume $(\mathrm{V})$, measured with a pair of calipers, was normalised to the day of irradiation $\left(\mathrm{V}_{0}\right)$ and response was evaluated as the time for the tumour to quadruple in volume $\left(\mathrm{T}_{4}\right)$. Rats were killed within 80 days of irradiation.

Data analysis. DCE data were analysed using a recent reference tissue model (Yankeelov et al, 2005) avoiding the need to directly measure the arterial input function (AIF). The model compares the contrast agent curves in a tissue of interest (tumour) to that of a 


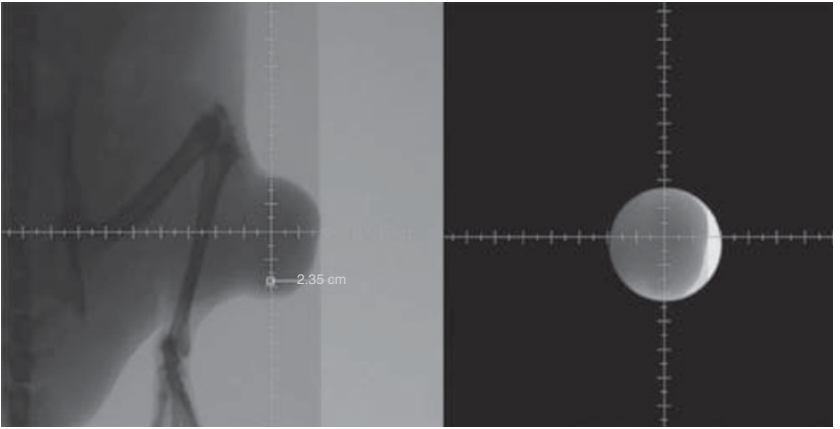

Figure 1. Image-guided radiation-beam delivery. Left: kV X-ray image showing tumour in hind leg. Right: radiograph showing the collimated field. $15 \mathrm{~Gy}$ (half dose) was delivered through a D-V field followed by 15 Gy from the opposite ( $V-D)$ side.

reference region (muscle). Using reported values for the volume transfer constant of muscle, $K^{\text {trans, }}{ }^{\mathrm{M}},\left(0.1 \mathrm{~min}^{-1}\right)$ and the extravascular-extracellular volume fraction, $v_{\mathrm{e}}, \mathrm{M},(0.1)$ in the muscle (Padhani, 2002, Yankeelov et al, 2005), it is possible to extract the $K^{\text {trans }}$ and $v_{\mathrm{e}}$ values for the tumour without knowledge of the AIF. In this model, the contrast concentration curve, $\mathrm{C}(\mathrm{t})$, in tumour tissue is given by (Yankeelov et al, 2005):

$$
\mathrm{C}(\mathrm{t})=R \times C_{\mathrm{M}}(t)+R \times\left[\frac{K^{\mathrm{trans}, \mathrm{M}}}{V_{\mathrm{e}, \mathrm{M}}}-\frac{K^{\mathrm{trans}}}{V_{\mathrm{e}}}\right] \times \int_{0}^{t}\left(C_{\mathrm{M}}\left(t^{\prime}\right) \times \exp \left(-\frac{K^{\mathrm{trans}}}{V_{\mathrm{e}}} \times\left(t-t^{\prime}\right)\right)\right) d t^{\prime}
$$

where, $\mathrm{R}=\left(K^{\text {trans }} / K^{\text {trans, }} \mathrm{M}\right)$ and $\mathrm{C}_{\mathrm{M}}(\mathrm{t})$ is the contrast agent concentration curve in muscle tissue. Analysis of BOLD and TOLD data (both semi-quantitative changes in signal intensity $(\Delta \mathrm{SI})$ and quantitative $\mathrm{R}_{1}$ and $\mathrm{R}_{2}{ }^{*}$ ) was described in detail previously (Hallac et al, 2014), and the results are compared with the new DCE data.

Statistics. Pearson correlation analysis was performed between the DCE-derived parameters and tumour growth delay. The significance of differences between Groups was established using analysis of variance. The statistical analysis was carried out by using Excel 14 (Microsoft, Redmond, WA, USA) software.

\section{RESULTS}

A baseline $T_{2}$-weighted $M R$ image slice is shown for a representative tumour (Figure 2A) together with heterogeneous contrast following administration of Gd-DTPA (Figure 2B). The $K^{\text {trans }}$ and $v_{\mathrm{e}}$ maps (Figure $2 \mathrm{C}$ and $\mathrm{D}$ ) were heterogeneous with most high values in the periphery. Mean $K^{\text {trans }}=0.11 \pm 0.05$ min $^{-1}$ and mean $v_{\mathrm{e}}=0.19 \pm 0.09$ ( 8 treated tumours).

Caliper measurement of tumour growth was obtained up to four times the initial tumour size or 80 days following irradiation. Untreated controls had the fastest growth rate with a $\mathrm{T}_{4}=8 \pm 1$ days. Irradiation caused significant tumour growth delay compared with non-irradiated tumours $(P<0.05)$ and $\mathrm{T}_{4}$ ranged from 28 to 75 days (Figure 3 ). For Group 2 (air) $\mathrm{T}_{4}$ ranged from 28 to 48 days. Overall there was no significant difference between $\mathrm{T}_{4}$ for air and oxygen groups, but two different responses were observed for Group 3 (oxygen) tumours. Some exhibited no significant benefit compared with air breathing $\left(\mathrm{T}_{4}=40-44\right.$ days; $P>0.4$ vs air). Others showed significantly enhanced tumour growth delay $\left(\mathrm{T}_{4}=70-75\right.$ days $(n=2) ; P<0.005$ vs air and $P<0.01$ vs the unresponsive tumours).

A strong correlation was observed between $v_{\mathrm{e}}$ and $\mathrm{T}_{4}$ for those tumours breathing air during irradiation (Group 2; $r^{2}>0.93$; $P<0.05$; Figure 4). Intriguingly a strong correlation remained if all tumours, irrespective of inhaled gas were combined (Groups $2+3$; $\left.r^{2}>0.72 ; P<0.001\right)$. For $K^{\text {trans }}$ there was strong correlation with $\mathrm{T}_{4}$

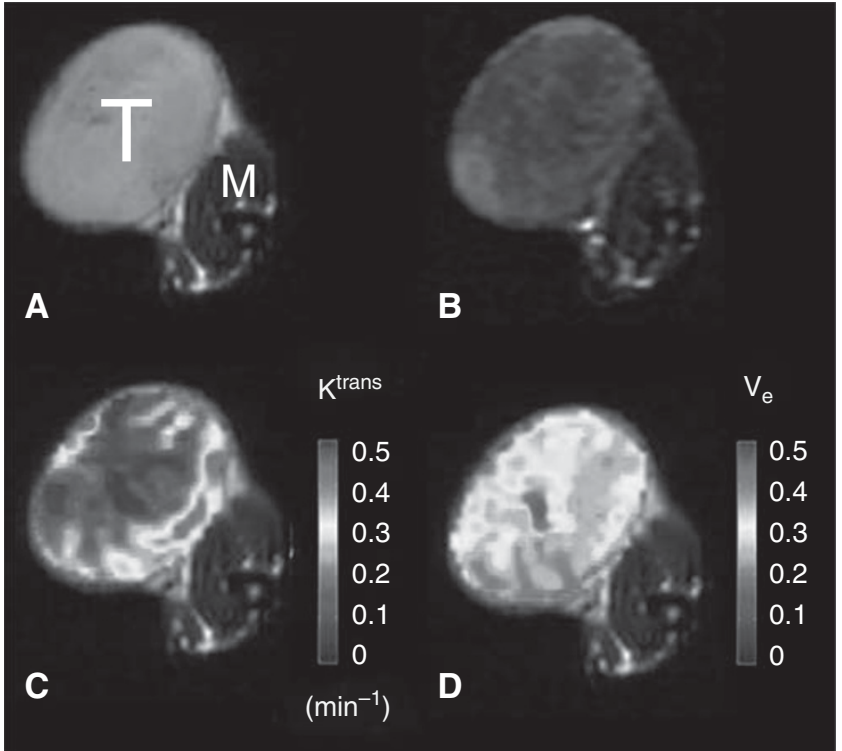

Figure 2. DCE MRI of subcutaneous R3327-AT1 tumour.

(A) High-resolution $\mathrm{T}_{2} \mathrm{~W}$ image showing tumour (T) and muscle (M). (B) Following administration of Gd-DTPA signal increase was observed throughout the tumour showing heterogeneous rates and amplitudes. (C) $K^{\text {trans }}$ and (D) $v_{e}$ maps overlaid on high-resolution $T_{2} W$ image of a small R3327-AT1 tumour with respect to Gd-DTPA infusion.

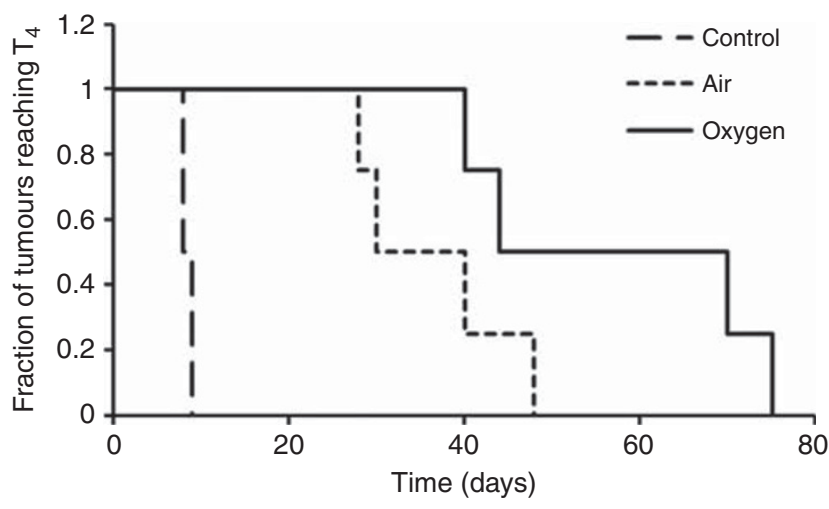

Figure 3. Kaplan-Meier plot showing the influence of radiation on Dunning prostate R3327-AT1 tumour growth. Fraction of AT1 tumours reaching $\mathrm{T}_{4}$ : non-irradiated control tumours vs single dose $30 \mathrm{~Gy}$, for rats breathing air or oxygen. Mean $\mathrm{T}_{4}$ Group $3\left(\mathrm{O}_{2}\right)=57$ days; Group 2 (air) $=36.5$ days, Group 1 (control) $=8.5$ days). Two-tailed comparison showed significant differences $(P<0.05)$ between irradiated tumours and control, though not between the irradiated groups. The Group 3 tumours which did not benefit from oxygen breathing behaved like those in Group 2.

for Group 2 tumours $\left(r^{2}>0.99 ; P<0.005\right)$, but this failed upon inclusion of Group 3 tumours $\left(r^{2}>0.3\right)$. There was a significant correlation between $v_{\mathrm{e}}$ and $K^{\text {trans }}$ for the eight irradiated tumours $\left(r^{2}>0.53 ; P<0.05\right)$.

The OE-MRI was analysed and published previously showing strong correlation between $\Delta \mathrm{SI}$ TOLD, $\Delta \mathrm{R}_{1}, \Delta \mathrm{T}_{1}$ and $\mathrm{T}_{4}$ (Hallac et al, 2014). No such correlations were observed between BOLD and $\mathrm{T}_{4}$. We have now examined relationships between OE-MRI and DCE, but in general, there was lack of correlation, although TOLD $(\% \Delta S I) v s v_{\mathrm{e}}$ showed $r^{2}>0.54(P<0.05)$ and $\mathrm{R}_{1} v s K^{\text {trans }}$ showed $r^{2}>0.59(P<0.05)$. 

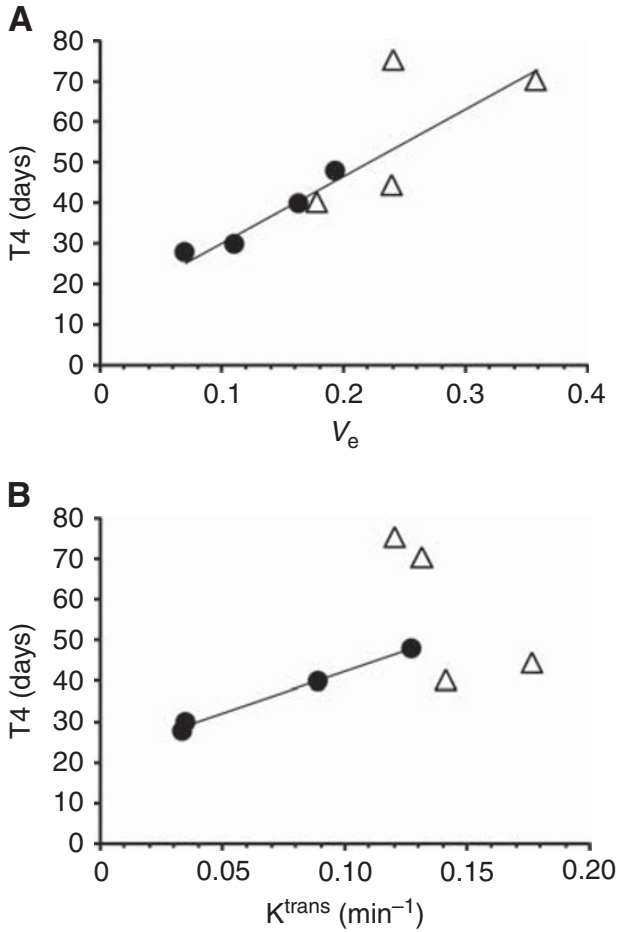

Figure 4. Tumour volume-quadrupling time $\left(\mathrm{T}_{4}\right)$ for AT1 tumours. (A) Correlation between $T_{4}$ and $v_{e}$ for those tumours irradiated during air $(\bullet, n=4)$ or oxygen $(\triangle, n=4)$ breathing. The best fit line indicated strong significant correlation $\left(r^{2}>0.72, P<0.05\right)$, which was even stronger for the Air group alone $\left(r^{2}>0.93, P<0.05\right)$. (B) Correlation between $\mathrm{T}_{4}$ and $K^{\mathrm{trans}}$. The best fit line indicated strong significant correlation for air-breathing group $\left(r^{2}>0.99, P<0.05\right.$, symbols as indicated in $\mathbf{A}$ ).

\section{DISCUSSION}

Distinct trends were observed between DCE MRI parameters and radiation treatment. Tumours exhibiting greater $v_{\mathrm{e}}$ values had longer tumour growth delay, irrespective of the inhaled gas. There was also a strong correlation between $K^{\text {trans }}$ and tumour growth delay for the air-breathing rats alone.

In an extensive series of studies Rofstad et al (2000) examined relationships between quantitative DCE parameters, hypoxia and radiation response (Gulliksrud et al, 2011; Egeland et al, 2012; Ovrebo et al, 2012). For cohorts of eight human melanoma lines, exhibiting varying degrees of radiobiological hypoxia when growing as xenografts in nude mice, Egeland et al (2012) reported robust correlations between $v_{\mathrm{e}}$ or $K^{\text {trans }}$ and radiobiological hypoxic fractions. Ovrebo et al (2012) found that $K^{\text {trans }}$ was significantly higher for individual radiation sensitive tumours, but $v_{\mathrm{e}}$ did not differ. Several of these studies examined tumour heterogeneity and sought to discard voxels corresponding to necrotic regions (Gulliksrud et al, 2011; Egeland et al, 2012). We examined tumour images of whole cross sections with no attempt to eliminate potential necrosis. None of the previous studies examined tumour growth delay, but rather the more traditional paired clonogenic survival assays for cells from tumours on anaesthetised mice irradiated, while breathing air or shortly after killing to induce hypoxia (Egeland et al, 2012; Ovrebo et al, 2012). Our approach was somewhat different using air or oxygen breathing to modulate hypoxia and assessing tumour growth delay over several weeks.

The magnitudes of $K^{\text {trans }}$ and $v_{\mathrm{e}}$ observed in this study lie in the ranges reported for various tumours such as human melanoma xenografts in mice (Gulliksrud et al, 2011; Ovrebo et al, 2012), and
Dunning prostate R3327-AT6.1 tumours in rats (Fan et al, 2010). We observed a significant correlation between $K^{\text {trans }}$ and $v_{\mathrm{e}}$ $\left(r^{2}>0.53\right)$. We used the reference tissue approach for analysis thereby avoiding the need for a direct measurement of AIF. Also, attempts to measure AIF directly may be confounded by blood vessel motion, flow and partial volume (Faranesh and Yankeelov, 2008). Validity of the reference region model is subject to interand intra-subject variability, but the method is widely used and accepted. A reference region model appears particularly suitable in this study, as the reference tissue (thigh muscle) is immediately adjacent to the tumour in the leg and should have similar blood supply. The reference tissue method avoids the need for a large supply blood vessel within the field of view and relaxes the need for extremely high temporal resolution. The search for prognostic biomarkers is complex and consensus may be difficult to attain, as reports consider many different disease sites, tumour sizes and therapies. Here, we examined response to single high-dose irradiation, essentially stereotactic ablation radiotherapy. The AT1 is reported to be quite radiation resistant and 30 Gy represents slightly less than half the $\mathrm{TCD}_{50}$ (Peschke et al, 1998, 2011). Tumour growth delay following single-dose irradiation should match the paired survival assays following single dose. Much irradiation follows a fractionated radiation therapy protocol and it was reported that the response of hepatocellular carcinoma could be predicted based on DCE characteristics after 2 weeks of therapy, but not baseline (Liang et al, 2007).

Viglianti et al (2009) found that pretreatment semi-quantitative DCE parameters were predictive of therapeutic response of canine soft tissue sarcoma to thermoradiotherapy. In many cases, semiquantitative approaches have been used to characterise tumours based on simple changes in $\mathrm{T}_{1^{-}}$or $\mathrm{T}_{2}$-weighted signal intensity, time to reach maximum signal or rates of signal change (Alonzi et al, 2007). The most widely used measurement is the initial area under the curve (IAUC) following infusion of contrast agent and we previously observed a wide range of values in the AT1 tumour, which correlated with BOLD signal response in some tumours (Jiang et al, 2004). We did find that semi-quantitative assessments were indicative of response to metronomic chemotherapy in AT1 tumours (Zhao et al, 2005).

The correlation between $v_{\mathrm{e}}$ and $\mathrm{T}_{4}$ might be explained by the larger extracellular extravascular space in the tumour, and therefore, less cellular density and oxygen consumption. Several factors affect the development of hypoxia in tumours including vascularity of the surrounding normal tissue, angiogenic factor secretion and the oxygen consumption of tumour cells (Moulder and Rockwell, 1984; Gulledge and Dewhirst, 1996). The AT1 tumour line is highly radiation resistant, anaplastic undifferentiated and variably hypoxic (Zhao et al, 2003; Hallac et al, 2014). In general large AT1 tumours are found to exhibit extensive hypoxia, whereas small tumours $\left(<2 \mathrm{~cm}^{3}\right)$ may be more or less-well oxygenated (Zhao et al, 2003; Hallac et al, 2014). Moreover, AT1 tumours, which are hypoxic, tend not to respond to oxygen-breathing gas (Hunjan et al, 2001; Bourke et al, 2007; Hallac et al, 2014).

The tumour growth delay observed here is in line with previous reports for this subline, notably $\mathrm{T}_{4}=11.6 \pm 1.3$ days and $56.0 \pm 8.3$ days for control and $30 \mathrm{~Gy}$, respectively (Peschke et al, 1998). That study made no attempt to assess or modulate tumour oxygenation and anaesthetised rats breathed $33 \% \mathrm{O}_{2}$ during irradiation. In another study, we reported that $\mathrm{T}_{2}$ (the time to double in volume) was a function of tumour $\mathrm{pO}_{2}$ based on ${ }^{19} \mathrm{~F}$ MRI relaxometry (Bourke et al, 2007). We attempted to modify levels of oxygenation and compared response with irradiation. There was no significant difference in $\mathrm{T}_{2}$ between tumours irradiated, while breathing air or oxygen and the study was terminated before $\mathrm{T}_{4}$. However, it was noted that those tumours in the air-breathing group continued growing monotonically, albeit somewhat more slowly, whereas 
those irradiated while breathing oxygen showed a peak volume around 10-15 days post irradiation and then tended to shrink. Those studies required direct injection of hexafluorobenzene reporter molecule, which is unlikely to become practical in patients and is inconvenient in pre-clinical studies.

In a more recent study, we had explored OE-MRI, as well as the DCE MRI presented here. Although various reports have indicated that BOLD response to oxygen-breathing challenge is related to changes in $\mathrm{pO}_{2}$ (Al-Hallaq et al, 1998; Baudelet and Gallez, 2002; Zhao et al, 2009), we found no specific correlation between $\mathrm{R}_{2}{ }^{*}$ or $\mathrm{T}_{2} \mathrm{~W}$-weighted signal response and tumour growth delay. We did however determine a correlation between TOLD $\left(\Delta \mathrm{R}_{1}\right.$ or $\mathrm{T}_{1} \mathrm{~W}$ signal responses) and $\mathrm{T}_{4}$ (Hallac et al, 2014). It was possible to stratify oxygen-breathing animals into responders and non-responders, as might be expected based on the dependence of $\mathrm{R}_{1}$ on $\mathrm{pO}_{2}$ (Edelman et al, 1996; Matsumoto et al, 2006; Beeman et al, 2015).

Group 3 showed no overall benefit from oxygen breathing compared with Group 2. However, a wide range of $\mathrm{T}_{4}$ (28-75 days) was observed and this was strongly correlated with $v_{\mathrm{e}}$ for all tumours. A similar correlation was seen for $\mathrm{T}_{4}$ and $K^{\text {trans }}$, although for Group 2 (air breathing) tumours only. Such observations could be of value in treatment planning. Moreover, those tumours which benefited significantly from oxygen breathing were identified based on a large TOLD response (Hallac et al, 2014) These results imply a role for combined OE- and DCE MRI.

Although this study is small, we believe it provides significant new data commensurate with previous reports. Notably, we now find that the relationships between $K^{\text {trans }}$ and $v_{\mathrm{e}} v s$ tumour growth delay in syngeneic rat tumours are consistent with clonogenic paired-cell surviving fractions in studies of multiple human melanoma xenografts in mice. Clearly, it will be important to develop this observation in additional tumour types and with respect to other therapy paradigms. DCE is more commonly used to identify and delineate tumours (Alonzi et al, 2007) and the potential for predicting radiation response represents a bonus.

\section{CONCLUSION}

A strong correlation was observed between $v_{\mathrm{e}}$ and tumour growth delay, irrespective of the inhaled gas during irradiation. No correlations were observed between OE-MRI and tumour growth delay and there were minimal correlations between OE-MRI and DCE parameters. Although contrast is part of most radiological examinations, it suggests that high temporal resolution DCE MRI could readily be implemented to provide predictive insight into response to radiation. It should be noted that this study used a single high-dose irradiation and this is becoming increasingly relevant as radiation oncology practice moves towards hypofractionated regimens such as stereotactic ablation radiotherapy (SABR).

\section{ACKNOWLEDGEMENTS}

This study was supported in part by grants from the NIH NCI (R01 CA139043) and infrastructure provided by the Southwestern Small Animal Imaging Research Program (SW-SAIRP) supported in part by $1 \mathrm{U} 24$ CA126608, Simmons Cancer Center (P30 CA142543) and AIRC (P41 EB015908). The small animal irradiator was purchased with funds from a Shared Instrumentation grant S10 RR028011.

\section{CONFLICT OF INTEREST}

The authors declare no conflict of interest.

\section{REFERENCES}

Al-Hallaq HA, River JN, Zamora M, Oikawa H, Karczmar GS (1998) Correlation of magnetic resonance and oxygen microelectrode measurements of carbogen-induced changes in tumor oxygenation. Int $J$ Radiat Oncol Biol Phys 41: 151-159.

Alonzi R, Padhani AR, Allen C (2007) Dynamic contrast enhanced MRI in prostate cancer. Eur J Radiol 63: 335-350.

Baudelet C, Gallez B (2002) How does blood oxygen level-dependent (BOLD) contrast correlate with oxygen partial pressure $\left(\mathrm{pO}_{2}\right)$ inside tumors? Magn Reson Med 48: 980-986.

Beeman SC, Shui Y-B, Perez-torres CJ, Engelbach JA, Ackerman JJH, Garbow JR (2015) O2-sensitive MRI distinguishes brain tumor vs radiation necrosis in murine models. Magn Reson Med; e-pub ahead of print 14 July 2015; doi:10.1002/mrm.25821.

Bourke VA, Zhao D, Gilio J, Chang C-H, Jiang L, Hahn EW, Mason RP (2007) Correlation of radiation response with tumor oxygenation in the dunning prostate R3327-AT1 tumor. Int J Radiat Oncol Biol Phys 67: 1179-1186.

Edelman RR, Hatabu H, Tadamura E, Li W, Prasad PV (1996) Noninvasive assessment of regional ventilation in the human lung using oxygen-enhanced magnetic resonance imaging. Nat Med 2: 1236-1239.

Egeland TAM, Gulliksrud K, Gaustad JV, Mathiesen B, Rofstad EK (2012) Dynamic contrast-enhanced-MRI of tumor hypoxia. Magn Reson Med 67: 519-530.

Evelhoch JL (1999) Key factors in the acquisition of contrast kinetic data for oncology. J Magn Reson Imaging 10: 254-259.

Eyal E, Degani H (2009) Model-based and model-free parametric analysis of breast dynamic-contrast-enhanced MRI. NMR Biomed 22: 40-53.

Falk S, Ward R, Bleehen N (1992) The influence of carbogen breathing on tumor tissue oxygenation in man evaluated by computerized $\mathrm{pO}_{2}$ histography. Br J Cancer 66: 919-924.

Fan X, Mustafi D, Zamora M, River JN, Foxley S, Karczmar GS (2010) Can DCEMRI assess the effect of green tea on the angiogenic properties of rodent prostate tumors? Physica Medica 26: 111-116.

Faranesh AZ, Yankeelov TE (2008) Incorporating a vascular term into a reference region model for the analysis of DCE-MRI data: a simulation study. Phys Med Biol 53: 2617-2631.

Fleming IN, Manavaki R, Blower PJ, West C, Williams KJ, Harris AL, Domarkas J, Lord S, Baldry C, Gilbert FJ (2015) Imaging tumour hypoxia with positron emission tomography. Br J Cancer 112: 238-250.

Fyles AW, Milosevic M, Wong R, Kavanagh MC, Pintilie M, Sun A, Chapman W, Levin W, Manchul L, Keane TJ, Hill RP (1998) Oxygenation predicts radiation response and survival in patients with cervix cancer. Radiother Oncol 48: 149-156.

Gulledge CJ, Dewhirst MW (1996) Tumor oxygenation: a matter of supply and demand. Anticancer Res 16: 741-749.

Gulliksrud K, Ovrebo KM, Mathiesen B, Rofstad EK (2011) Differentiation between hypoxic and non-hypoxic experimental tumors by dynamic contrast-enhanced magnetic resonance imaging. Radiother Oncol 98: 360-364.

Hallac RR, Zhou H, Pidikiti R, Song K, Stojadinovic S, Zhao D, Solberg T, Peschke P, Mason RP (2014) Correlations of noninvasive BOLD and TOLD MRI with $\mathrm{pO}_{2}$ and relevance to tumor radiation response. Magn Reson Med 71: 1863-1873.

Horsman MR, Mortensen LS, Petersen JB, Busk M, Overgaard J (2012) Imaging hypoxia to improve radiotherapy outcome. Nat Rev Clin Oncol 9: 674-687.

Hunjan S, Zhao D, Constantinescu A, Hahn EW, Antich PP, Mason RP (2001) Tumor Oximetry: demonstration of an enhanced dynamic mapping procedure using fluorine-19 echo planar magnetic resonance imaging in the Dunning prostate R3327-AT1 rat tumor. Int J Radiat Oncol Biol Phys 49: 1097-1108.

Isaacs JT, Isaac WB, Feitz WFJ, Scheres J (1986) Establishment and characterization of 7 Dunning prostate cancer cell lines and their use in developing methods for predicting metastatic ability of prostate cancer. Prostate 9: 261-281.

Jiang L, Zhao D, Constantinescu A, Mason RP (2004) Comparison of BOLD contrast and Gd-DTPA dynamic contrast enhanced imaging in rat prostate tumor. Magn Reson Med 51: 953-960.

Kaanders JHAM, Bussink J, Van Der Kogel AJ (2004) Clinical studies of hypoxia modification in radiotherapy. Sem Radiat Oncol 14: 233-240. 
Le QT, Chen E, Salim A, Cao HB, Kong CS, Whyte R, Donington J, Cannon W, Wakelee H, Tibshirani R, Mitchell JD, Richardson D, O'Byrne KJ, Koong AC, Giaccia AJ (2006) An evaluation of tumor oxygenation and gene expression in patients with early stage non-small cell lung cancers. Clin Cancer Res 12: 1507-1514.

Liang PC, Ch'ang HJ, Hsu C, Tseng SS, Shih TTF, Wu Liu T (2007) Dynamic MRI signals in the second week of radiotherapy relate to treatment outcomes of hepatocellular carcinoma: a preliminary result. Liver Int 27 : 516-528.

Loncaster JA, Carrington BM, Sykes JR, Jones AP, Todd SM, Cooper R, Buckley DL, Davidson SE, Logue JP, Hunter RD, West CML (2002) Prediction of radiotherapy outcome using dynamic contrast enhanced MRI of carcinoma of the cervix. Int J Radiat Oncol Biol Phys 54: 759-767.

Lyng H, Vorren AO, Sundfor K, Taksdal I, Lien HH, Kaalhus O, Rofstad EK (2001) Assessment of tumor oxygenation in human cervical carcinoma by use of dynamic Gd-DTPA-enhanced MR imaging. J Magn Reson Imaging 14: $750-756$.

Ma CM, Coffey CW, Dewerd LA, Liu C, Nath R, Seltzer SM, Seuntjens JP (2001) AAPM protocol for 40-300 kV X-ray beam dosimetry in radiotherapy and radiobiology. Med Phys 28: 868-893.

Mason RP, Zhao D, Pacheco-Torres J, Cui W, Kodibagkar VD, Gulaka PK, Hao G, Thorpe P, Hahn EW, Peschke P (2010) Multimodality imaging of hypoxia in preclinical settings. QJ Nucl Med Mol Imaging 54 259-280.

Matsumoto K, Bernardo M, Subramanian S, Choyke P, Mitchell JB, Krishna MC, Lizak MJ (2006) MR assessment of changes of tumor in response to hyperbaric oxygen treatment. Magn Reson Med 56: 240-246.

Mayr NA, Wang JZ, Zhang D, Montebello JF, Grecula JC, Lo SS, Fowler JM, Yuh WTC (2009) Synergistic effects of hemoglobin and tumor perfusion on tumor control and survival in cervical cancer. Int J Radiat Oncol Biol Phys 74: 1513-1521.

Moulder J, Rockwell S (1984) Hypoxic fractions of solid tumors, experimental technique, methods of analysis and survey of existing data. Int J Radiat Oncol Biol Phys 10: 695-712.

Movsas B, Chapman JD, Hanlon AL, Horwitz EM, Greenberg RE, Stobbe C, Hanks GE, Pollack A (2002) Hypoxic prostate/muscle Po-2 ratio predicts for biochemical failure in patients with prostate cancer: Preliminary findings. Urology 60: 634-639.

Overgaard J (2007) Hypoxic radiosensitization: adored and ignored. J Clin Oncol 25: 4066-4074.

Ovrebo KM, Hompland T, Mathiesen B, Rofstad EK (2012) Assessment of hypoxia and radiation response in intramuscular experimental tumors by dynamic contrast-enhanced magnetic resonance imaging. Radiother Oncol 102: $429-435$.

Padhani AR (2002) Dynamic contrast-enhanced MRI in clinical oncology: current status and future directions. J Magn Reson Imaging 16: 407-422.

Peschke P, Hahn EW, Wenz F, Lohr F, Braunschweig F, Wolber G, Zuna I, Wannenmacher M (1998) Differential sensitivity of three sublines of the rat Dunning prostate tumor system R3327 to radiation and/or local tumor hyperthermia. Radiat Res 150: 423-430.

Peschke P, Karger CP, Scholz M, Debus J, Huber PE (2011) Relative biological effectiveness of carbon ions for local tumor control of a radioresistant prostate carcinoma in the rat. Int J Radiat Oncol Biol Phys 79: 239-246.

Rofstad EK, Sundfor K, Lyng H, Trope CG (2000) Hypoxia-induced treatment failure in advanced squamous cell carcinoma of the uterine cervix is primarily due to hypoxia-induced radiation resistance rather than hypoxia-induced metastasis. Br J Cancer 83: 354-359.

Sheridan MT, West CM, Cooper RA, Stratford IJ, Logue JP, Davidson SE, Hunter RD (2000) Pretreatment apoptosis in carcinoma of the cervix correlates with changes in tumour oxygenation during radiotherapy. $\mathrm{Br} J$ Cancer 82: 1177-1182.

Tatum JL, Kelloff GJ, Gillies RJ, Arbeit JM, Brown JM, Chao KSC, Chapman JD, Eckelman WC, Fyles AW, Giaccia AJ, Hill RP, Koch CJ, Krishna MC, Krohn KA, Lewis JS, Mason RP, Melillo G, Padhani AR, Powis G, Rajendran JG, Reba R, Robinson SP, Semenza GL, Swartz HM, Vaupel P, Yang D, Croft B, Hoffman J, Liu GY, Stone H, Sullivan D (2006) Hypoxia: Importance in tumor biology, noninvasive measurement by imaging, and value of its measurement in the management of cancer therapy. Int J Radiat Biol 82: 699-757.

Tofts PS (1997) Modeling tracer kinetics in dynamic Gd-DTPA MR imaging. $J$ Magn Reson Imaging 7: 91-101.

Tofts PS, Brix G, Buckley DL, Evelhoch JL, Henderson E, Knopp MV, Larsson HB, Lee TY, Mayr NA, Parker GJ, Port RE, Taylor J, Weisskoff RM (1999) Estimating kinetic parameters from dynamic contrast-enhanced T(1)-weighted MRI of a diffusable tracer: standardized quantities and symbols. J Magn Reson Imaging 10: 223-223.

Vaupel P, Mayer A (2007) Hypoxia in cancer: significance and impact on clinical outcome. Cancer Metast Rev 26: 225-239.

Viglianti BL, Lora-Michiels M, Poulson JM, Lan L, Yu DH, Sanders L, Craciunescu O, Vujaskovic Z, Thrall DE, Macfall J, Charles CH, Wong T, Dewhirst MW (2009) Dynamic contrast-enhanced magnetic resonance imaging as a predictor of clinical outcome in canine spontaneous soft tissue sarcomas treated with thermoradiotherapy. Clin Cancer Res 15: 4993-5001.

Wang Z, Su M-Y, Nalcioglu O (2002) Applications of dynamic contrast enhanced mri in oncology: measurement of tumor oxygen tension. Technol Cancer Res Treat 1: 29-38.

Wilson WR, Hay MP (2011) Targeting hypoxia in cancer therapy. Nat Rev Cancer 11: 393-410.

Workman P, Aboagye EO, Balkwill F, Balmain A, Bruder G, Chaplin DJ, Double JA, Everitt J, Farningham DAH, Glennie MJ, Kelland LR, Robinson V, Stratford IJ, Tozer GM, Watson S, Wedge SR, Eccles SA, Navaratnam V, Ryder S. Committee of the National Cancer Research Institute (2010) Guidelines for the welfare and use of animals in cancer research. Br J Cancer 102: 1555-1577.

Yankeelov TE, Luci JJ, Lepage M, Li R, Debusk L, Lin PC, Price RR, Gore JC (2005) Quantitative pharmacokinetic analysis of DCE-MRI data without an arterial input function: a reference region model. Magn Reson Imaging 23: 519-529.

Zahra MA, Hollingsworth KG, Sala E, Lomas DJ, Tan LT (2007) Dynamic contrast-enhanced MRI as a predictor of tumour response to radiotherapy. Lancet Oncol 8: 63-74.

Zhao D, Jiang L, Hahn EW, Mason RP (2005) Continuous low-dose (Metronomic) chemotherapy on rat prostate tumors evaluated using MRI in vivo and comparison with histology. Neoplasia 7: 678-687.

Zhao D, Jiang L, Hahn EW, Mason RP (2009) Comparison of ${ }^{1} \mathrm{H}$ blood oxygen level-dependent (BOLD) and ${ }^{19} \mathrm{~F}$ MRI to investigate tumor oxygenation. Magn Reson Med 62: 357-364.

Zhao D, Ran S, Constantinescu A, Hahn EW, Mason RP (2003) Tumor oxygen dynamics: correlation of in vivo MRI with histological findings. Neoplasia 5: 308-318.

This work is published under the standard license to publish agreement. After 12 months the work will become freely available and the license terms will switch to a Creative Commons AttributionNonCommercial-Share Alike 4.0 Unported License. 